\title{
Ein Fall von beiderseitigem centralem Scotom mit pathologisch-anatomischem Befund.
}

Beitrag zur Kenntniss des Verlaufs der Maculafasern im N. options, Chiasma und Tractus optious.

Voin

Dr. A. Vossius,

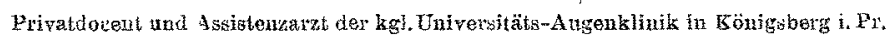

Hierzu Tafel VI.

Ueber den Verluuf der Sehnervenfasem im menschlichen Chiasma standen sich bisher zwei Ansichten gegenüber, die der vollständigen Kreuzung, die in der Neuzeit von Biesiadecki (1), Michel (2) und Mandelstamm (3) besonders lebhaft vertheidigt wurde, und die der Partialkreazung, die schon ron Johinnes Müller (4) ausgesproehen, durch Hannover's (匚) eingeliende Arbeiten bestätigt und später im Wesentlichen von Henle (6) u. A. acceptirt wurde. In den letzten 10 Jahren hat diese Ansicht gegenuber den Michel'sehen Resultaten ihren eifrigsten Vertreter in v. Gudden (7) and seit der Publikation seiner unfangreichen Arbeiten wohl allgemeine Anerkemung gefunden. Auch Stilling (8), dec mit Loupe und Zelzupfungsuadel an Holzessigpräparaten den Faserverlauf im Chiasma, Tractus und N. opticus untersuchte, hat sich in sciner umfangreichen Monographie entschieden für die Partialkreazung naw Maassgabe seiner Beobachtungen 
anscesprochen. Was num durch die Cutersuchung normaler Chiasmen wegen der äusserst dichten und innigen VerHechtung der sehr feinen Nervenfasem nur sehr schwer, ja fast unmöglich zu ermitteln scheint, hat v. Gudden bei Thieren auf dem Wege des Experimentes und an wenschlichen Präparaten, in denen die Pathologie den Weg des Experiments eingeschlagen hatte, auf's Untrüglichste klar gelegt. Die Frage der Partialkreuznng ist larnach für den Menschen und die höheren Wirbelthiere, die ein mehr minder grosses gemeinschaftliches Gesichtsfeld haben, absolut sicher entschieden, ausserdem ist anch festgesetzt, dass das ungekreuzte Bündel um so grösser ist, je grösser das gemeinschaftliche Gebiet des Gesichtiofolles ausfällt.

Gleichzeitig mit $\vee$. Gudden gelangte zu dem Resultate der Partialkreuzung Nicati (9), aber auf anderem Wege, inden er bei Thieren das Chiasma in der Mittellinie durchtrennte, während $G$. das eine Auge junger Thiere bald nach der Geburt ennoleirte und die Folgen des Eingriffs nach mehrmonatlichem Zuwarten durch die mikroskopische Untersuchung des Chiasma und der Tractus ermittelte.

Munk (10) griff die Sehcentren direkt duroh Anlegung vou Defekten in der Hirnrinde der Occipitallappen bei Hunden und Affen an, und beobachtete nach einseitiger Operation gleichseitige homonyme Hemianopsie, ein Beweis dafur, dass jeder Traetus für beide Angen Nervenfasern vom Centrum fuhrt, die sich in identischen Netzhant. bezirken verbreiten, dass also jeder Opticusstamm aus beiden Tractus seine Fasern erhält.

Bei dem Menschen hat die Pathologie auch fur diese letzteren Thierexperimente in bereits zahlreichen Krankheitsfällen mit Sectionsbefund entsprechende Resultate geliefert, ja das über ähnlichen, aber zuvor mnerklärlichen Fällen herrschende Dunkel ist erst, seitdem Munk seine Beobachtungen bekannt gemacht hat, vollständig gelichtet. So 
ist nur noch neuerdings von Marchand (11) ein bereits sehr lange zuvor zur Section gelrommener, schr interessanter und reiner Fall von Läsion der Occipitalrinde veröffentlicht, der früher die Obducenten stutzig gemacht hatte, weil sich bei der Autopsie kein Erklärungsgrund für die bei Lebzeiten beobachtete homonyme bilaterale Hemianopsie in den Thalamis oder den weiter nach dem Bulbus zu gelegenen nervösen Abschnitten des Sehapparates - wie eigentlich erwartet war - gefunden hatte. In dieser Arbeit theilte Marchand noch einen genau mikroskopisch untersuchten Fall von einseitiger glaucomatöser Opticusatrophie mit, in dem an Serienschnitten durch das Chiasma und beide Tractus der atrophische Process verfolgt war. Derselbe schloss sich in seinem Verlauf eng an die früher von Baumgarten*), Gudden, Woinow (12), Purtscher (13) veröffentlichten Fälle einseitiger Opticusatrophie an; gekreuztes und ungekrenztes Bündel waren isolirt atrophirt, ihre Lage in dem Chiasnla und Tractus entsprach vollständig den Angaben v. Gudden's, nach denen das ungekreuzte Bündel im Chiasma und Tractus lateral liegt, im Chiasma das gekreuzte, dem andern Tractus entstammende überkreuzt, und im Sehnervenstamm selbst an der medialen. Seite verläuft. Im Tractas ist jedoch die Grenze beider Bündel keineswegs absolut scharf; stellenweise fanden sich bei einseitigen Atrophien, so auch in Marchand's Fall, in dem einen atrophisehen Büudel zerstreut wohl erhaltene Nervenfasergruppen, dio dem anderen unbetheiligten Faseikel angehörten. Kellermann (14) ist aber entschieden zu weit gegangen mit seiner Angabe, dass im Tractus überhaupt keine fasciculare Anordnung der Sehnervenfasem existire, sondern dass alle Nervenfasern innig und regellos mit einander durchflochten seien.

*) Centralblatt $f$. d. med. Wissensch, 1878. 
Für die Frage mah dom Verlauf und der Lage des gekreuzten and ungekreuzten Bündels in Tractus und Chiasma ist damn noch sehr interessant und lehrreich ein bisher einzig dastehender Fall, den Ganser (15) kürzlich mitgotheilt hat. Das ungekrenzte Bündel wurde dargestellt von einem isolirt verlaufenden Nervenstrang, der auf der ventralen Seite cles Tractus auflag, mehr medial in Corpus geniculatum externum entsprang, am Tractus bis dicht an's Chiasma medial verlief, hier einen Bogen nach der lateralen Seite des Chiasma beschrieb, im Chiasma und Sehnervenstamm lateral blieb and schliesslich $34 \mathrm{~mm}$ vor dem Bulbus in die Scheiden des Opticus sich einsenkte, um sich weiterhin nicht mehr isolirt verfolgen zu lassen.

Ueber den Verlauf und die Lage der zu beiden Faseikeln gehorigen Gruppen von Nervenfasern in dom eigentlichen Sohnervenstamm ist bisher fast gar nichts Nüheres ermittelt. Leber (16) latte in einer früheren Arbeit über retrobulbäre Neuritis angegeben, dass die Nervenfasern im Opticus rom Chiasma bis zum Bulbus ziemlich gestreckt verliefen, doch ist nach Kellermann's und Samelsohn's (17) Beobachtungen, denen sich auch die meinigen ansonliessen, ein Weclisel des gegenseitigen Lageverhältnisses der rerschiedenen Faserbündel ziemlich sicher, für die Maculafasem nahm Leber denselben auch in seiner später erschienenen Abhandlung im Handbuch von GraefeSämisch an.

Dass dio Opticusfasern von der Papille in die Retina in ridiärer Richtung ausstrahlen, ist durch Liebreich (18) uphthalmoskopisch festgestalt und darch Michel's (19) anatomische Untersuchungen bestatigt; die zur Macula lutea verlaufenden Sehnervenfasern, die ich weiterhin ler Kürze halber Maculafasem nennen will, ziehen darnach ziemlich genau horizontal in die temporale Falfte der Retina, die den äusseren Abschnitt der letzteren versorgenden Fasern umkreisen die Maculafasern wach oben 
und unten in grösseren Bögen und verlanfen erst hinter der Macularegion radiär weiter.

Leber hatte bereits im Jahre 1869 die Vermuthung ansgesprochen und später im Handbuch noch näher begrïndet, dass die Maculafasern im Sehnervenkopf sämmtlich bei einander und zwar in dessen äusserer Halfte nahe den Opticusseheiden gelegen sein, und dass die zur übrigen äusseren Retinaparthie ziehenden Fasern die axialen Bündel bilden müssten, woraus folge, dass die Nervenfasern einfach in die Netzhaut umbiegen und in der Papille sich nicht von innen nach aussen durchkreuzen, was bei dem entgegengesetzten Lageverhältniss der Fall sein müsste. Leber nahm nun bei den Fallen von centralem Scotom ohne ophthalmoskopischen Befund an der Macula lutea eine Erkrankung des Opticusstammes selbst an, die er als retrobulbäre Neuritis genauer beschrieb; bei derselben sollten speciell die an die Scheide aussen anstossenden Fasergruppen des opticus afficirt resp. atrophirt sein, weil sich im weiteren Verlauf, gelegentlich aber auch schon im Anfang der klinischen Beobachtung eine atrophisehe Verfärbung der äusseren Papillenhälfte bemerkbar mache, in der nach Liebreich's und Michel's Angaben gerade die zim: Macula ziehenden Nervenfasern gelegen seien.

Schwalbe (20) äussert sich hinsichtlich der Ausbreitung der Nervenfasern in der Retina allgemein dahin, "dass die ausseren Bündel des Sehnerven auch am weitesten nach aussen in der Nervenfaserschicht zu liegen kommen, sich also früher in letztere einsenken als die centralen Nervenfaserbündel, welche zunächst auf der inneren Oberfläche der Retina ihre Ausbreitung finden."

Magnus (21) trat Leber's Ansicht entschieden entgegen. In seiner Arbeit über die Sehnervenblutungen zeigte er, dass sich bei denselben vorzugsweise in der Gegend der Macula und nächsten Nachbarschaft der Papille eine grauweisse Tribung des Angenlintergrundes befände, 
die nach seiner Ansicht auf fettig-degenerativem Zerfall der hier gelegenen Fasern infolge der mangelnden Blutzufuhr beruht. Die Blutung erfolgt aus den Centralgefüssen, es müssen also zunächst die in der Axe um dieselben gelegenen Nervenfasergruppen unter der Compression durch das ausgetretene Blut leiden; die Affection derselhen markirt sich ophthalmoskopisch in der Trübung des Augenhintergrundes an der Macula und der nach aussen von der Papille gelegenen Netzhautparthie. Die Veränderungen an der Macula finden sieh nun aber auch bei Blutungen in die Sehnervenscheiden, die nicht ans den Centralgefässen entstehen, bei denen also die axialen Fasern nicht zunächst direkt betroffen werden - schon hieraus ist ersichtlich, dass Magnus' Annahme, die Maculafasern verliefen in der Axe des Sehnervenstammes, nicht in allen Fallen zur richtigen Erklärung der vorhandenen ophthalmoskopischen Frscheinungen führt; er gesteht denn auch selbst im weiteren Verlauf seiner Abhandlung die Unzulänglichkeit seiner Hypothese für manche Krankheitsfalle zu.

Wilbrand (22) reprodueirt in seiner Arbeit über Neuritis axialis die Förster'sche Ansicht, die mit der Magnus'schen Annahme vollständig identisch ist; er erklärt sich dabin, dass die Maculafasem, die dem Fascieulus cruciatus entstammen, nach der Art der Erkrankung und nach Maassgabe der Gesichtsfelder nahe beisammen liegen müssen, und dass die die Peripherie der Netzhaut, versorgenden Fasern im Sehnervenstamm peripher, die für lie Macula bestimmten central gelegen seien.

Alle diese Hypothesen uber die Lage der Maculafasern standen indessen auf schwachen Füssen, so lange der pathologisch-anatomische Befund fehlte. Derselbe entzog sich bisher merkwürdigerweise immer den Augen der Ophthalmologen, obwohl die Krankheitsfalle mit centralem Scotom, bei denen keine Veränderungen des Augenlintergrundes in der Maculagegend als Ursache nachweisbar 
sind, der Herd des Leidens als eine retrobulbäre Neuritis im Sinne Leber's aufgefasst werden muss, unter den Sehnervenaffectionen am häufigsten sind; die Ursache für den mangelnden anatomisehen Befund liegt allein in der Langwierigkeit des Leidens, das für die Sehkraft relativ gutartig genannt werden muss, und in der Machtlosigkeit der Therapie selbst bei monatelanger äratlicher Behandlung.

Die Deductionen Leber's über die Lage der Maculafasern in der Papille wurden auf's Glänzendste als zutreffend gekennzeichnet in einem neuerdings von Samelsohn genau untersuchten Fall von centralem Seotom ohne ophthalmoskopischen Befund, den er auch bei Lebzeiten längere Zeit hindurch beobachtet und früher trotz hartnăckiger Negation des Abusus spirituosorum et nicotini zur Kategorie der Intoxikationsamblyopieen gerechnet hatte. Nur insofern stellte sieh der Befund anders heraus, wie von Leber erwartet war, als die Maculafasern ibre periphere Lagerung nach dem Foramen opticum zu allmählig mit der axialen vertausehen, was aber mit der Argumentation, aus welcher die periphere Lagerung in der Nähe des Auges gesehlossen wurde, gar nicht im Widerspruch steht, und worüber natürlich nur direkte Untersuchungen Aufschluss geben konnten. Der Kranke von Samelsohn war mehrere Jahre nach seiner ersten Vorstellung an einem Herzleiden im Kölner Bürgerspital verstorben, und nur durch einen Zufall hatte Samelsohn die Sehnerven und Bulbi zur Untersuchung bekommen. Er fand bei derselben in beiden Opticis vom Foramen optieum an bis zur Papille den gleichen Process an durchweg symmetrischen Stellen etablirt, in der Gegend des Foramen opticum die Zeichen einer in narbige Schrumpfung mit Atrophie der Nervenfasern ausgegangenen Entzündung des Sehnerven, welche genau die centralen Abschnitte der Optici befallen hatte, während die periphere Ringzone normal war. Die atrophisehe Stelle nälerte sich weiter abwarts mehr dem 
Iateralen Rande des Sehnervenquerschnitts und nahm schliesslich dicht am Bulbus den äusseren Sector desselben in Gestalt eines Keiles ein, dessen Basis nach der Sehnervenscheide, dessen Spitze nach den Centralgefässen zu lag; die Ausdehnung des atrophischen Sectors betrug olngefähr $1 / 3$ des Querschnitts-Flächeninhalts. Während im Allgemeinen von dem primixen Herd im Foramen opticum aus eine descendirende Atrophie sich entwickelt batte, war an der Bintrittsstelle der Centralgefässe in den Sehnerven eine Rxacerbation des entzündlichen Procasses zu constatiren. Chiasma und Tractus, sowie der intracranielle $A b-$ schnitt des Opticus war beiderseits normal. Die Papille und Retina zeigte sich entspreehend dem Sektor temporalwärts atrophisch, die Atrophie umfasste die Taser- und Ganglienzellenschicht. Nach diesem mikroskopischen Befund lag also eine partielle Neuritis dem bei Lebzeiten beobachteten centralen Scotom a Grunde; die Maculafasem waren durch den Process za Grunde gegangen and im Sehnervenstamme isolirt dargestellt. Ihre Lage ist also im Canalis opticus in der Axe des Sehnervenquerschnits, wahreud die periphere Zone das excentrische Sehen vermittelt. Weiter nach dem Bulbus zu rückt das axiale Bündel immer mehr lateralwärts und crreicht schliesslich ginz den temporalen Rand des Sehnerven. Mit dem Lagewechsel verändert sich auch die Form des Bündels, sie geht aus der des Cylinders in die eines Keils über; Ursache der Formverinterung ist der Eintrict der Centralgefässe, welche die zuvor peripheren Bündel vor sich her nach dem Centrum schieben und die mehr centralen Gruppen nach der Peripherie drängen. Da mun die Maculafasurn sowohl dem nicht gekreuzten wie dem gekreuzten Bündel entstammen, so nimmt Samelsobn an, dass sich vor dex Papille von dem nach Gudden's Angabe medial Fonlaufenden nicht gekreuzten Fascikel die Maculafasern nozweigen und zu den dem gekrenten zugehörigen tem- 
poralen Maeulafasern gesellen, um mit ihnen in der äussern Papillenhälfte vereint in mehr minder horizontaler Richtung direkt nach der Macula lutea zu ziehen.

In einem bald darauf von Nettleship (23) publicirten Falle von Amblyopia centralis bei einem Diabetiker war bei der mikroskopischen Untersuchung in dem dem Bulbus dicht anliegenden exstirpirten Opticusstumpf ebenfalls ein atrophischer Sektor gefunden, dessen Lage und Ausdehnung so vollständig dem von Samelsohn beschriebenen Bilde glich, dass N. eine genauere Beschreibung desselben beinahe für überflüssig erklärte.

Ich bin in der glücklichen Lage, in den folgenden Zeilen einen dem eben kurz scizzirten ähnlichen Befund wiederzugeben. Den betreffenden Fall verdanke ich der Güte des Herrn Professor Leber; das zugehörige Chiasma mit einem Stück des demselben anhaftenden rechten Tractus opticus - der linke war bei der Section dicht am Chiasma abgeschnitten - sowie die beiden Opticusstämme mit den hinteren Bulbusabschnitten waren, in Müller'scher Flüssigkeit gehärtet, Herrn Professor Leber von Herrn Professor Westphal zu wissenschaftlicher Verwerthung übersandt. Die Retina war so gefaltet und brüchig, dass nur der unmittelbar an die Papille angrenzende Abschnitt zur Untersuchung verwerthet werden konnte. Nach genügender Entwässerung und Nachhärtung in starkem Alkohol wurde an die Bearbeitung der Präparate gegangen.

Zuvor will ich noch die betreffende Krankengeschichte mittheilen; dieselbe ist bereits bei einer andern Gelegenheit ron Binswanger (24) in dem Archiv für Psychiatrie publicirt, dürfte an dieser Stelle aber den Fachgenossen entgangen sein, so dass eine Wiedergabe derselben der Vollständigkeit wegen am Platze erscheint.

Wilhelm Wolfsdorf, 48 Jahre alt, wurde am 30. Okt. 1880 in die Charité-Abtheilung von Professor Westpha] aufF. Grabfe's Archip fitr Ophthalmologte XXVIII. 3. 
genommen und starb am 25. November 1880. Er stammte ans gesunder Familie, hatte 3 mal gastrisehes Fieher uberstanden, war sonst nie krank gewesen; kein Trauma, keine Lues; mässiger Abusus spiritnosorum. Vor 3 Jahren hatte er bemerlkt, dass seine Augen schwächer wurden, so dass er nach 3 Monaten Gedrucktes und Geschriebenes nicht mehr lesen konnte. Er wurde auf der Augenstation dor Charité behandelt und wurde damals die Diagnose Scotoma centrale (Professor Schweigger) festgestellt. Die Sehschärfe soll sich später wieder allmählig gebessert haben.

Im October $1880 \mathrm{kam}$ er in die Nervenabtheilung wegen eines Cerebralleidens, von dem er die ersten Spuren im März zuvor bemerkt hatte: Hemiplegie des rechten Armes unter Bewusstseinsverlust und Zuckungen. Darauf wiederholte Anfälle von Bewusstseinsverlust mit krampfhaften Zuckungen der rechten Oberextremität. Mitte September 3 solcher Anfälle an einem Tage, darauf motorische Schwäche des r. Armes ohne deutliche Lähmung, dann der $\mathrm{r}$. Gesichtshälfte und des r. Beines. Am 26. Oktober deutliche Parese der r. Extremitäten und des Facialis in der NIundpartie. Keine Störung der vegetativen Functionen, kein Kopfschmerz, Schwindel oder Erbrechen, nur schlechter Schlaf; keine Abnahme des Gedächtnisses, das erst nach dem letaten Anfall etwas schlechter geworden, gleichzeitig Sprachstörung, die sich nach Angabe der Fran weiterhin verschlimmerte.

Aus dem Status praesens bei der Aufnahme in die Nervenklinik hebe ich hervor, dass der mittelgrosse, gut genährte Kranke guten Schlaf und Appetit, ein freies Sensorium hatte. Der schädel war ohne ăusserliche Abnormitäten und lokalen Schmerz, die linke Pupille etwas kleiner als die rechte, die sehlechter reagirte als die linke. Das Gesichtsfeld schien für einfachere Untersuchungen nicht beschränkt, die Bewegungen der Bulbi waren frei, die ophthalmoskopische Untersuchung ergab normale Verhältnisse. Gehör, Geruch, Geschmack waren normal. Facialis in Mund-, Wangen- und Augenzweigen paretisch, Sprache mit Andeutung von Aphasie. Totale motorische Lähmung der r. oberen, Parese der r. unteren Extremität, ohne Sensibilätsstörungen. - Die Parese des rechten Beines und Facialis wandelte sich später in totale Paralyse $\mathrm{um}$, die Aphasie wurde ansgesprochoner. Am 25. November Exitus. 
Die am 26. November vorgenommene Obduetion ergab folgenden Befund: Grosses, ziemlich schweres Schädeldach, Diploe sehr blutreich, Dura mater straif gespannt, ihr Längssinus völlig leer. Innenfläche beiderseits vollkommen glatt und glänzend, Gefässe sehr stark gefüllt. Nach der Entfernung der Dura sieht man links durch die Pia mater hindurch an einer Stelle, welche dem Vereinigungspunht der zwoiten Stirnwindung mit der vorderen Centralwindung entspricht, einen dreieckigen 2 em langen, mit seiner Basis ebenso breiten gelben Herd, innerhalb dessen die Pia vollkommen adhärent ist. Der übrige Theil der Pia ist sehwach odematös und lässt sich ohne Sehwierigkeiten ablösen. Die ganze linke Hemisphäre ist stark abgeplattet, die Windungen sind entschieden verbreitert, von blassem Aussehen, allgemein stärker durchfenchtet. Die vordere Centralwindung ist in ihrem obern Drittheil in eine etwa $4 \mathrm{~cm}$ lange breiig weiche Masse von graugelber Färbung umgewandelt, an deren vorderem und unterem Ende der vorher erwähnte mit der Pia adhärente gelbe Herd sitzt. Am oberen medianen Ende des Erweichungsherdes greift derselbe noch $1 \mathrm{~cm}$ weit auf den hintern Theil der obern Stirnwindung über. Die hintere Centralwindung ist an ihrem vordern Rande wie zugeschärft und legt sich wie eine glatte Falte über den hintern Theil des Erweichungsherdes. Mit dem Skalpelstiele lïsst sie sich von dem Herde bequem abdrängen, wodurch die Tiefe der Centralfurche frei gelegt wird. Die Frweichnng greift nirgends anf die hintere Centralwindung über, die Tiefe der Centralfurche intact. Der Klappdeckel stark abgeplattet, die Inselwindungen völlig frei, ebenso die mediane Fläche der Centralwindungen. Nirgends weitere Herderkrankungen auf der Oberfläche beider Homisphären. - Ein Frontalsehnitt durch die Mitte der erweichten Partie (vordere Centralwindung) zeigt die Rinde daselbst ganz zerstört, in eine gelblich-braune und graugelbe breiige Masse umgewandelt; an der Begrenzungslinie der grauen und weissen Substanz scheint noch ein ganz schmaler Streifen sehr anämischer grauer Substanz erhalten zu sein, das unten liegende Marklager, etwa $1,5 \mathrm{~cm}$ breit, makroskopiseh intact. - Darunter trifft man auf eine wallnussgrosse Höhle, gefüllt mit brännlicher klarer Flüssigkeit. Die Wand der Höhle völlig glatt, wie ron einer Schleimbant bekleidet, die Innenfläche sehr gefässreich, zum Theil nit brännlichen Pigmentablagerungen. Nach unten und innen erstreckt 
sich dieso Höhle einerseits bis dicht an die äussere Kapsel, andererseits an das Dach des Ventrikels und den Balken, jedoch ist dieselbe aberall von dem Gebiet der Stammganglien durch einen Streifen makroskopisch normal erscheinender Marksubstanz getrennt. Weitere Herderkrankungen im Gehirn nicht vorhanden. Medulla oblongata nnd Rückenmark makroskopisch frei.

Die mikroskopische Untersuchung des Erweichungsherdes zeigte zahlreiche, meist pigmenthaltige Körnchenzellen, Hämatoidinkrystalle, amorphes Blutpigment, körnige Detritusmassen, keine Nervenelemente. Im frisch untersuchten Rückenmark keine Erkrankung der Vorder- oder Seitenstränge, auch die innere Kapsel frei von Körnchenzellen. Die Untersuchung des in Müller'scher Lösung gehärteten Pückenmarks bestätigte dieses Ergebniss, indem überall, sowohl bei der Behandlung mit Kali, als anch geeigneten Farbungsmethoden normale Verhältnisse sich vorfanden.

Herr Professor Westphal hatte die Güte, mir über das frühere Augenleiden noch folgende Notizen zukommen zu lassen, für die ich ihm hiermit bestens danke:

Vor 3 Jahren wurde Patient an Seotoma centrale in der Augenstation behandelt, er war aber schon früher schwachsichtig. Damals (1878) wurde er nach Behandlung mit Inunctionen, Schwitzen, Strychnininjectionen als gebessert entlassen. $\mathrm{S} \mathrm{R}_{\mathrm{*}}=\frac{2,5}{24}, \mathrm{~L} .=\frac{3}{24}$; mit convex 10 wurde beiderseits 0,75 in 0,20 gelesen. Ans dem Status praesens hob er noch besonders hervor, dass keine Abweichung des intraocularen Befundes, sowie des Scotoms bestand. Der Kranke wurde von Professor W. klinisch vorgestellt und bei der Demonstration besonders betont, dass das centrale Scotom für die Diagnose nicht zu verwerthen sei, da es schon so lange bestehe und seine Ursachen bisher unbekannt seien. Leider fehlt mir über die Ausdehnung des Scotoms sowie über das sonstige Gesichtsfeld etwas Genaneres; die hierdurch bedingte kleine Lücke des Falles dürfte indessen für die Verwerthung des Befundes kraum storrend sein, da meine Resultate der mikroskopischen Untersuchung so vollkommen mit denen Samelsohn's, ron dessen Kranken genane Gesiehtsfelder vor- 
lagen, übereinstimmen, dass ich mit Nottleship fast eine genawere Beschreibung für überflüssig erklären könnto.

Aeusserlich liess sich an den Sehnerven, dem Chiasma und rechten Tractus keine erhebliche Anomalie constatiren, dahingegen zeigten sich auf Querschnitten durch die gedachten Gebilde erhebliche Veränderungen, die schon durch die Farbenuntersehiede sehr in die Augen fielen. Beiderseits erwies sich der Opticus dicht am Bulbus auf dem Querschnitt nicht rund, sondern abgeplattet (Fig. 2) an der äussern Seite, namentlich unten aussen, so dass seine Gestalt mehr einem schrägen Oval glich. Dieser abgeplatteten Contourpartie entsprach eine sectorenförmige weisse Stelle, deren Basis an die Sehnervenscheiden grenzte, deren Spitze nach der Mitte des Querschnitts sah und, wie bei Loupenvergrösserung ersichtlich wurde, an der Stelle der Centralgefässe endigte, welche dem Rande des Quersehnitts hier erheblich näher lagen als in den anderen Richtungen und in der Norm. Dieser weisse Sector, der sich gegen die übrige normal aussehende, durch die Chromwirkung grünlich verfärbte Nervenmasse des Querschnitts wesentlich abhob, nahm den untern äussern Quadranten ein. Der grösste Durchmesser des Quersehnitts war der Höhendurchmesser; or mass $1.3^{1 / 2}$, r. etwas über $3^{1 / 4} \mathrm{~mm}$, der kleinste Durchmesser - schräg von oben innen nach unten aussen gemessen, so dass derselbe den Sector halbirte - betrug 1 . und r. $2^{1 / 2} \mathrm{~mm}$; es war also eine starke Differenz in beiden Richtungen gegen die normalen Verhältnisse zu constatiren, unter denen die gedachten Durchmesser bei kreisrundem Quersehnitt ca. $4 \mathrm{~mm}$ messen. Der Zwischenscheidenraum erschien erheblich aufgelockert, die äussere Scheide in der Gegend des Sectors weit von dem eigentlichen Nervenstamm abgedrängt und dentlich gefaltet. Die innere Scheide umzog als gleichmässig schmaler hellgrauer Streifen die ganze Peripherie des Nervenquerschnitts. 
8 mm rom Bulbus entfernt zeigle der letztere beiderseits wiederun erhebliche Farbenunterschiede, die sich. auch hier wesentlich auf den untern äussern Quadranten beschränkten. Die Gestalt der weissen Partie war nicht mehr die eines Sectors, sie grenzte an die Peripherie mit breiter Basis (Fig. 3) und sandte nach der Mitte zu einzelne mehr minder spitze Fortsätze, am Rande war dio Centralvene, in der Mitte die Arterie deutlich sichtbar. Die Form des Querschnitts war die eines Oblongs, dor Querdurchmesser - $3 \mathrm{~mm}$ - erheblich verringert, der Höhendurchmesser - $3 \frac{1}{2} \mathrm{~mm}$ - auch etwas kleiner als normal. Die äussere Scheide erschien auch hier wieder rom eigentlichen Sehnerven an der helleren Stelle abgedrängt und gefaltet, lag ihm im Uebrigen aber normal an.

$16 \mathrm{~mm}$ rom Bulbus entfernt, vor dem Eintritt der Centralgefässe, näherte sich die Gestalt des Querschnitts wehr der rundlichen Form (Fig. 4), doch liess sich auch lier eine Verringerung dex Durchmesser constatiren, hier betrugen vertikaler und horizontaler Durchmesser $3^{1 / 4} \mathrm{~mm}$. Der Scheidenraum war nicht so aufgelockert, die äussere Soheide lag dem Nerv überall ziemlich eng an. Die hollo Stelle hatte wieder eine annähernd keilförmige Gestalt, doch lag hier die Basis mehr in der Witte, die Spitze des Keiles hart an der Peripherie, der pialen Scheide dicht an. Die helle Partie zeigte an ihrer Basis keine lineare Begrenzung, es fanden sich in derselben einzelne dunklere Punkte, die bei Loupenvergrösserung sich als normal gefäbte Nervensubstanz auswiesen. Der Keil lag auch hior wieder mehr im untern äussern Quadranten.

Auf einem $24 \mathrm{~mm}$ rom Bulbus entfernt gelegenen Querschnitt (Fig. 5) befand sich die helle Stelle excentrisch mehr in der äusseren temporalen Halfte und hatte annähernd eine halbmondförmige Gestalt; sie lag von einer normal gefärbten Zone ringsum umgeben, dieselbe war nach der melialen Seite zu breiter als nach der lateralen, 
nach oben und unten zu ziemlich gleich breit. Nach der Mitte des Querschnitts zu befand sich der Loupenvergrosserung nach eine Uebergangszone, in der atrophische und nichtatrophische Nervensubstanz vermiseht war. Auffallend war die Gestaltsveränderung des Quersehnitts, derselbe war besonders im Querdurchmesser verkleinert und bildete ein stehendes Oval, das am äusseren Rande etwas vorgebuckelt, am innern Rande an der entsprechenden Stelle leicht eingezogen erschien. Die Scheide lag dem Nerv hier eng an.

Bin Querschnitt durch den Opticus dicht hinter dem Foramen opticum cerebralwärts, kenntlich an dem Fehlen der duralen Scheide (Fig. 6), hatte querovale, von oben nach unten abgeplattete Gestalt, war $5 \mathrm{~mm}$ breit, $3 \frac{1}{4} \mathrm{~mm}$ hoch. Die inmere Halfte sah höher als die äussere aus, zwischen beiden befand sich am untern und obern Rande eine seichte Einbuchtung. Fast genau in der Mitte des Querschnitts, mehr dem obern Rande genähert, lag hier ein querovaler, heller Strich, der oben aussen fast den Rand des Querschnitts berührte, in Uebrigen von einer normal aussehenden, in allen Richtungen gleich breiten Zone umgeben war; der helle Streifen sthien im Allgemeinen eine grössere Ausdehnung zu haben als auf den übrigen Querschnitten.

Etwa $1 \mathrm{~cm}$ vor dem Chiasma (Fig. 7) erschienen beide Optioi abgeplattet, $5 \mathrm{~mm}$ breit, $3 \mathrm{~mm}$ hoch; in der Mitte des sonst grünlich gefärbten Querschnitts fiel eine weisse spindelförmige Stelle auf, die unten und zu den Seiten von anscheinend normalem Gewebe umgeben war. Von der Mitte des oberen Randes zog schräg nach innen an jenem weissen Strich vorbei ein hellgelber Streifen, der, in der Nähe des medialen Querschnittsrandes angelangt, sich dendritisch verzweigte, die Verzweigungen strahlten in mehr minder welligem Verlauf divergent nach dem obern innern, innern und untern inneren Rand des 
Querschnitts aus. Schon durch diesen Verlauf markirten sie sich als bindegewebige Septen innerhalb des Opticusstammes, die von dem Piaüberzug herzurühren sehienen. Aehnliche helle Striche strahlten auch nach allen Richtungen von dem centralen weissen Fleok aus und exreichten, soweit bei Loupenvergrösserung sichtbar war, theils die Ränder des Schnitts, theils endeten sie blind in der Ungebung. Diese helle Stelle behielt ihre Lage durch das ganze letzte Stück des Opticus bis dicht an's Chiasma heran bei.

In Folgendem will ich noch eine kurze Uebersicht der Durchmesserverhältnisse von diesen verseliedenen Querschnitten der Optici geben:

\begin{tabular}{|c|c|c|c|c|c|c|}
\hline & & & & & H. D. & V. D. \\
\hline & & & & & $\mathrm{mm}$ & $\mathrm{mm}$ \\
\hline Dicht am Bulbas . . & , & & . & & $21 / 2$ & $3^{1 / 2}$ \\
\hline $8 \mathrm{~mm}$ vom Bulbus . & 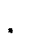 & & & . & 3 & $31 / 2$ \\
\hline $16 \mathrm{~mm}$ vom Bulbus.. & 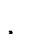 & & 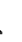 & . & $31 / 4$ & $31 / 4$ \\
\hline $24 \mathrm{~mm}$ vom Bulbus . . & . & & & . & 3 & $31 / 2$ \\
\hline Hinter dem Canal. opt. & . & • & • & & 5 & $3^{3} / 4$ \\
\hline 1. cm vor dem Chiasma & . & & . & & 5 & 3 \\
\hline
\end{tabular}
messer.

H. D. bedentet den horizontalen, V. D. den vertikalen Dureh-

Zur mikroskopischen Untersuchung wurde das Chiasma mit dem $r$. Tractus and beiden Optieis in eine nach Schiefferdecker's Vorschrift gemachte Celloidinlösung eingebertet. Das Verfahren, welches ich in Rostock kennen gelernt hatte, und das Sohiefferdecker (25) inzwischen selbst genauer besclrieben hat, fand ich für den vorliegenden Zweek um so geeigneter, weil die Einbettungsmasse in nicht zu grosser Dicke beim Erstarren ihre Durchsichtigkeit bewalirt, und deshalb die Controle aber: 
die richtige Führung lor beabsichligten Schnitte stets offen bleibt. Die Masse schneidet sich aus freier Hand and mit dem Milkrotom vorzüglich und hat neben der Durchsichtigkeit gerade für leichtbrüchige Gebilde, wie z. B. die Retina, den Vorzug, dass man sie bei regularer Nachbehandlung der Schnitte nicht zu entfernen brancht; die einzelnen Theile eines Gebildes also vollständig in situ erhalten bleiben können - bekannt ist ja, wie leicht die Opticusscheiden bei den sonst üblichen Behandlungsweisen an Sehnitten abfallen und der Untersuchung im Zusammenhang mit dem Nerven entgehen. - Um Wiederholungen zu vermeiden, will ich gleich erwähnen, dass die Schnitte in Alauncarmin (Grenacher) resp. Hämatoxylin gefärbt wurden; diese Behandlung genugte zur Unterscheidung der atrophischen von den nieht atrophisehen Partieen vollständig. Des Ueberfinsses halber behandelte ich auch einzelne Schnitte mit Goldchlorid, ohne dass indessen dadurch mein Einblick in die gedachten Verhältnisse mehr gefördert worde als durch die beiden andern Farbemittel.

Chiasma und Tractus der $\mathrm{x}$. Seite wurden in eine fortIaufende Serie von Schnitten mit dem Mikrotom zerlegt. Ich schildere hier nur zunächst das makroskopische Aussehen der Schnitte aus der grossen Serie von Frontalschnitten, weil die dazwischenliegenden keine Differenz gegen einander darboten. Auf Schnitten aus der vordern Hälfte des Chiasma vor Beginn des Recessus opticus (26) ergab sich, dass am dorsalen Rande ein heller schmaler Streifen verlief, der der Commissura ansata Hannover's angehörte, darunter markirte sich eine ziemlich ausgedehnte gelbliche Stelle, die sehr erheblich gegen die übrige Partie des Schnitts auffiel. Die gelbe Stelle hatte ohngefähr Bohnenform (Fig. 8), verlief mit ihren convexen Rand dorsalwärts dicht unterhalb des hellen Streifens der Commissura ansata, ihr concaver Rand lag centralwärts obngefähr in der Mitte des Schnitts, die Anschwellungen be- 
fanden sich zienlich central in den Seitentheilen des Chiasmaschnitts, die sthmalste Stelle in dessen Mitte. Bei Loupenvergrösserung sah man deutlich von einer Seite zur andern hinüberziehen abwechselnd helle und dunkle Striche, die letzteren reichlicher in der an die normal gefarbte ventrale Zone des Querschnitts angrenzenden Partie, während die gelbliche Stelle dicht unterhalb der Commissura ansata eine gleichmässige helle Farbe der Schnittfläche und keine dunklen Züge zeigte. In der Gegend des Recessus opticus (Fig. 9) hob sich die obere und untere graue Begrenzungsschicht desselben scharf gegen die eigentliche Chiasmamasse ab, die unmittelbar an jene Schicht anstossend einen hellgelben Streifen enthielt, der mehr Bisquitform hatte und mit seinen Anschwellungen genau in der Mitte der Seitentheile, mit seiner Finschnurung in der Mitte des Querschnitts lag. In diesem hellen Streifen liessen sich mit der Loupe keine dmikelgrünen Striche wahmehmen. Die Breite des Chiasmafrontalschnittes aus der vordern Hälfte betrug $12 \mathrm{~mm}$ - gegen $14 \mathrm{~mm}$ nach Luschka (27) und 13-14 mm nach Michel (28), die Höhe in der Mitte $3 \mathrm{~mm}$ (nach Luschka 6, nach Michel 5-6 $\mathrm{mm}$ unter normalen Verbältnissen). Nach dem Tractus zu verschmälerte sich die dünne Stelle zwischen den seitlichen Anschwellungen immer mehr, schwand schliesslich ganz.

Im Anfangstheil des r. Tractus, dessen Querdurchnesser $5 \mathrm{~mm}$ und dessen vertikaler Durchmesser $3 \mathrm{~mm}$ betrug, markirte sich im obern äussern Quadranten eine helle Stelle, die schräg vom obem Rande, dort wo der Tractus mit den darüber befindlichen Hirnabschnitten fest rerwachsen ist, nach der Mitte des Querschnitts zu verlief and sich noch etwas in den untern äussern Quadranten hineinerstreckte, ausserdem ein hellgelber Strich längs des ganzen untern Randes (Fig. 10 a und b). Die erstere entsprach hinsichtlich ihrer Lage nach den übereinstimmenden 
Befunden Baumgarten's, Gudden's, Purtscher's, Ganser's und Marchand's dem ungelrreuzten, der helle Strich am untern Rande dem gekreuzten Fascikel des Tractus. Durch alle weiteren Sehnitte des letzteren bis ans Ende des vorhandenen Abschnitts waren diese beiden hellen Stellen nachweisbar.

Der hintere Bulbusabschnitt wurde jederseits in eine obere und untere Hälfte getheilt durch einen Schnitt, der genau durch die Mitte des hellen Keils im untern äussern Quadranten, also nicht ganz in der Horizontalen verlief. Auf diesem Querschnitt zeigte sich nun, dass im äussem Drittheil des intrascleralen Opticusabschnitts bis etwa all die Centralgefässe heran ein heller Strich längs der Pial= scheide sich befand, der sich durch die Lamina cribrosa in die Papille hinein erstreckte.

Durch beide Nerven liess sich mithin von der Papille bis ins Chiasma und in den erhaltenen rechten Tractusabschnitt eino helle Zone erkennen, die in der Papille und dem an den Bulbus angrenzenden Opticustheil genau den untern änssern Quadranten einnahm, weiter aufwärts unter Vertauschung der Lage von Basis und Spitze des Keils mehr nach der Mitte des Querschnits verlief, dann ein liegendes Oval im Centrum des in Canalis opticus befindlichen Sehnervenabschnitts darstellte, und central blieb durch den ganzen intracraniellen Abschnitt des Optiens und des Chiasma, während im Tractus eine Sonderung in zwei Herde eintrat, von denen der eine im Bereich des Fascieulus cruciatus, der andere in dem des noneruciatus lag.

Bei der Beschreibung der mikroskopischen Bilder werde ich mich an die in der Abbilkung gegebenen Hauptschnitte halten, weil in den dazwischen gelegenen Serienschnitten sich continumich derselbe Befund wiederholte. Der der Fig. 10 entsprechende Tractusquersehnitt (Fig. 14) zeigte in der angrenzenden Partie des Tuber eincreum (T.c.) keine wesentliche Anomalie, dio Gefässe lagen in grossen 
vacuolenartigen Hohlräumen, welche stellenweise mit einer amorphen, fibrinartigen, äusserst lockeren und durehsichtigen Masse ausgefüllt waren; die Wände der Arterien waren etwas verdickt, die der Venen normal, die Verdickung bestand sowohl an den grösseren als an den kleineren Gefässen, deren Lumen mit Blutkörperchen dicht erfüllt war. Amyloidkörperchen der verschiedensten Grösse und Form lagen in der amorphen Masse, die die grossen Gefässlücken erfüllten, um die letzteren und in der periphersten an die Pia angrenzenden feinkörnig-faserigen Schicht des Tuber und der Substantia perforata anterior, sowie des Ganglion optieum, dessen Ganglienzellen keine Abnormitäten zeigten. Die Amyloidkörperchen waren ausserordentlich zahlreich und nahmen ganze Striche der Tractusquerschinite ein. Auch in der periphersten schmalen Schicht des eigentlichen Tractus fanden sie sich in grosser Reichlichkeit. Sie zeigten rundliche, ovale, bisquitförmige Gestalt, schwankten ziemlich exheblich in ihrer Grösse and zeigten vielfach eine blasse Hülle um den intensiv gefärbten Inhalt. Sie glichen vollkommen den von Leber (29), Treitel (30) and Gudden beschriebenen Formen, einen Kern konnte ich an ihnen nicht constatiren. Die helle Stelle im obern Abschnitt und am untern Rande des Tractus hatte bei Carmin- und Hämatoxylinfärbung an sämmtlichen Schnitten eine gleichmässige, ziemlich intensive Färbung angenommen, erschien auffallend durchsichtig glasig gegen die undurchsichtige, ungefärbte, grünlich bleibende Umgebung der normalen Tractusgebiete, in denen sich nur die Neuroglia- und Gefässkerne, sowie die sie begleitenden spärlichen Bindegewebszüge gefarbt hatten, während dazwischen die in ihrer Grösse wechselnden Nervenfaserquerschnitte ihr normales Aussehen beibehalten hatten. Die diffus und sehr intensiv gefärbte Partie, die rom obern Rande der Tractusquerschnitte nach ihrer Mitte zu sich ausbreitete, erwies 
sich als eine faserige Gewebsmasse, in der an Zahl nicht vermehrte Kerne, einzelne Amyloidkörperchen, aber keine normalen Nervenfasern mehr nachweishar waren, die letzteren waren hier vollständig atrophiseh zu Grunde gegangen. Ebenso liessen sich in der am untern Rande beschriebenen Zone keine normalen Nervenfaserquerschnitte auffinden, sie bestand aus einem feinkörnig-faserigen Gewebe, wie es sonst in atrophischen Sehnerven beobachtet ist. Die Kerne waren auch hier nicht vermehrt, einzelne Amyloidkörperchen in dem atrophischen Bezirk wie in der sonst normalen Umgebung vorhanden. Beide atrophische Zonen zeigten auf allen Traetusquersehnitten bis zum Chiasma das gleiche Verhalten.

Frontalschnitte des letzteren aus der hinteren Hälfte, entsprechend der Fig. 9, erwiesen den hier vom Schnitt getroffenen Recessus opticus nicht erweitert, mit normalem Eipithel ausgekleidet, die ihn umgebende graue Substanz zeigte im Allgemeinen nichts Abnormes, zu den Seiten des Recessus hoben sich schon makroskopisch grosse und zahlreiche Lücken ab, in denen Gefässquerschnitte lagen, welche das Lumen der Hohlräume keineswegs ganz ausfüllten, verdickte Wandung hatten und zum Theil in eine feinfaserige, durchsichtige Masse eingebettet waren, die den Eindruck geronnener Lymphe machte. In der Umgebung dieser Vacuolen war das Gewebe bis an den Rand der Schnitte, sowie ihre ganze Peripherie dicht erfüllt mit Amyloidkörperchen, die an einzelnen Stellen so reichlich waren, dass man nichts ron dem Gewebe, in welchem sie eingebettet waren, bemerken konnte. Der bereits makroskopisch sichtbare hellgelbe Streifen hatte sich mit Carmin und Hämatoxylin intensiv gefärbt, zeigte keine Spur normaler Nervenfasern, dagegen sich wirr durchflechtende feinfaserige gefürbte Züge, die schräg von einer Seite zur andem zogen, atropische Nervenfasern darstellten, getrennt durch fein punktirtes, ebenfalls gefarbtes Gewebe, welches 
atrophischem Nervengewebe glich und den im Querschnitt getroffenen atrophischen Nervenfasern entsprach. Die wirr durchflochtenen Züge fanden sich nur in der Mitte des hellen durchsichtigen Streifons, nicht an den Rändern, in der Umgebung hatte das Gewebe den Charakter normalen Nervengewebes, die Fasern waren ungefarbt geblieben, theils im Querschnitt, theils schräg, theils paraliel zu ihrem Verlauf auf eine kurze Streeke getroffen, an den Qnerseluitten komte man die Axencylinder dentlich unterscheiden. In dem unterhalb des hellen Striches gelegenen normalen Abschnitt der Chiasmaquerschnitte fand sich das wirre dichte Geflecht der normalen Sehnervenfasern, das Michel und Gudden vorzuglich abgebildet haben und so dicht war, dass eine Orientirung über den Verlauf einzelner Fasern nur sehr unvollkommen gelang. Sie waren theils quer, theils schräg, theils auf längere Strecken parallel zu ihrem Verlauf getroffen, Kerne und Gefässe weder hier noch in dem atrophischen Strich vermehrt, am Rande vereinzelte Corpuscula amylacea.

Auf Sehnitten weiter nach rom im Chiasma - entsprechend Fig. 8 - ungefalir aus der Mitte der vorderen Halfte zwischen vorderer Commissur und Recessus opticns sah der helle Herd makroskopisch etwas grösser aus, bei stärkerer Vergrösserung liess sich indessen feststellen, dass in schräger Richtung von der einen zur andem Seite commissurenartig verlaufende sehr feine, gefärbte, also atrophische Nervenfaserzïge abwechselten mit Gruppen ungefärbter Nervenfaserzüge von normalem Durchmesser, die die gleiche Verlaufsrichtung hatten; dazwischen fanden sich ausserdem noch atrophisch und normal aussehende im Querschnitt resp. schräg getroffene Nervenfasern, dic am Rande des atrophischen Bezirks ein wirres Durcheinander bildeten. Zwischen den atrophischen Fasern lagen vereinzelt grössere und kleinere Gefässquerschnitte und Capillaren: die grösseren Gefisse hatten durchweg 
verdickte Wandungen. Mit den Gefässen drangen in das Innere des Chiasma septenartige Fortsetzungszüge des Pian̈berzugs, kenntlich an den zahlreichen, stark bräunlich pigmentirten stern- and spindelformigen kernhaltigen Zellen. Kerne und Gefässe waren weder in der atrophischen noch in der normalen Partie in vermehrter Zahl vorhanden.

In dem unmittelbar vor dem Chiasma gelegenen intracraniellen Abschnitte der Opticusstämme lag der atrophische Herd central (Fig. 7), in demselben endigten von allen Seiten der Peripherie eindringende Fortsätze der $\mathrm{Pia}$, in denen sich reichliche Corpuscula amylacea befanden, einzelne enthielten feinere Capillarästchen, welche an der eigenthümlichen Anordnung der Kerne kenntlich waren. Vom obern Rande her zog nach der Mitte der Querschnitte 7,11 ein breiter bindegewebiger dicht mit Pigmentzellen durchsetzter Streifen, der neben einem grösseren Gefässe auffallend viel Corpuscula amylacea enthielt. Der centrale Herd hatte sich mit den Färbemitteln intensiv und gleichmässig tingirt, in demselben liess sich keine normale, sondern nur fein punktirte, atrophische Nervensubstanz nachweisen, wathrend in der ganzen umgebenden Ringzone normale Gruppen ron Faserquerschnitten mit wohl erbaltener Markscheide und deutlich tingirtem normalem Axencylinder lagen. Gefässe und Kerne waren auf den Querschnitten nicht in vermehrter Zahl vorhanden.

Eine auffallende Veränderung zeigte das Bild der Sehnervenschnitte dicht vor dem Canalis opticus, wo bereits die Scheiden ausgebildet waren und der Nervenstamm aus der platten, bandartigen in eine mehr rundliche Form uberzugehen anfing. Hier war der Charakter der hellen atrophischen Stelle und des ganzen Querschnittsbildes wesentlich anders. Die helle Zone zeigte eine so dichte Kerninfiltration (Fig. 13), dass nur stellenweise eine Andeutung einer Zwisehensubstanz kenntlich war. Nerveu- 
fasern waren nirgends nachweisbar, die Septenzüge, erheblich verbreitert, bestanden aus welligen Bindegewebsfasern, die in ihren netzartigen, erheblich verkleinerten Hohlrüumen keine normalen Nervenfaserquerschnitte, sondern feinkönig-faseriges, sehr fein punktirtes Gewebe onthielten, wie es für atrophische Sehnerven charakteristisch ist. Die Septen waren rollständig unregelmässig verzerrt, zeigten auch in der mehr normalen Umgebung des atrophischen centralen Herdes keine ganz regelmässige Anordnung, erst an der Peripherie der Schnitte waren sie in normaler Breite und Lage nachweisbar. Hier erst lagen innerhalb der Septen normale Gruppen von Nervenfaserquerschnitten; in der Umgebung des centralen, durchsichtigen, atrophischen Herdes fand sich eine Uebergangszone, in der innerhalb der Septen atrophische and normale Nervenfaserquerschnitte bei einander lagen. In den Knotenpunkten mehrerer Septen waren meist mehrere Querschnitte kleiner Gefässe mit verdiekter, kernreicher Wandung, die sämmtlich mit Blutkorperchen dicht erfüllt waren. Einzelne verbreiterte und sehr kern- und gefässreiche Septen reichten oben aussen bis dicht an den Piaüberzug heran, der normale Breite und keine Keminfiltration zeigte. Amyloidliörperchen fanden sich sowohl in dem atrophisehen Herd als auch in der Peripherie der Schnitte an den Septen und in deren Maschenräumen.

Das mikroskopische Bild der hellen Zone in drm Sehnervenstamm zeigte nur bis zur Papille abwärts stets die gleichen Figenschaften. Der normale Septenbau war verwischt, die Septen erschienen unregelmässig verzerrt und verbreitert, in ihren Knotenpunkten befanden sich reichliche Gefässdurchschnitte, ferner waren die Kerne an Zahl sehr erheblich vermehrt. Innerhalb dieses Herdes liessen sich bis zur Papille normale Nerventaserquerschnitte nicht mehr constatiren, das Bild des die Maschenrüume erfüllenden Gewehes glich vollständig dem atrophischer 
Nervensubstanz, es stellte dar eine äusserst kernreiche, feinfasrige, sehr fein punktirte Masse, die sehr durchseheinend war und sich mit Carmin und Hümatoxylin zum Untersehied gegen die normale Umgebung sehr stark tingirt hatte. Amyloidkörperchen waren in dem ganzen intraorbitalen Absehnitt des Sehnerven in erheblich geringerer Zahl nachweisbar. An der Fintrittsstelle der Gefässe erschien der centrale Bindegewebsstrang verbreitert, der atrophische Herd nicht scharf abgegrenzt, sondern durch eine schmale Uebergangszone, innerhalb deren atrophische und nichtatrophische Nervenbündel untermiseht lagen, von der ganz normalen Zone getrennt. Im Ganzen entsprach aber der atrophische Theil des Querschnitts dicht am Bulbus ohngefähr dem 4. Theil desselben; alle Querschnitte vom Canalis opticus bis zum Bulbus zeigten auch innerhalb der Septen der äusserlich normalen Partie eine erhebliche Vermehrung der Kerne und einen grösseren Gefässreichthum (efr. Fig. 12 und 13).

Ein Querschnitt durch die Papille und den intraseleralen Absehnitt des Sehnerven (Fig. 15) liess erkennen, dass in dem äusseren Dritttheil desselben die Nervensubstanz vollständig atrophiseh und dureh éin sehr kernreiches Gewebe ersetzt war, in dem sich ausserdem noch reichliche Capillaren nachweisen liessen. Die atrophische Zone reichte bis an die Centralgefässe, die von einem breiten Bindegewebsstreifen umgeben waren und verdiekte, kernreiche Wandungen hatten. Ibr Lumen war dieht erfüllt mit Blutkörperchen. Die Septen des normalen Nervenabschnitts enthielten ebenfalls an Zahl sehr vermehrte Kerne. Der atrophische Strich liess sich bis in die temporale Hälfte der Papillen verfolgen, der entsprechende Abschnitt der Netzhant war verdünnt, die Gangliensehicht in dem dicht an die Papille anstossenden Bezirk der Retina entschieden schwächer entwickelt als auf der nasalen Seite, wo die Ganglienzellen in mehreren Reihen angeordnet

v. Graefe's Arohiv fir Ophthamologie, XXVIII. 3 , 
waren. Sowohl in dem markhaltigen intraseleralen, wie in dem marklosen papillaren Theil des Sehnerven waren Amyloidkörperchen vorhanden, die sich auch in dem an die.Papille angrenzenden nasalen and temporalen Netzhantstuck vorfanden. Auch die Papille zeigte einen enormen Kernreichthum.

Um nun einen kurzen Ueberblick über vorliegenden Fall zu geben, so hatte sich bei einem mässigen Potator 3 Jahre vor seinem Tode, der nnmittelbar an den Folgen einer Herderkrankung der linken Grosshirnhemisphäre eingetreten war, eine beiderseitige hochgradige centrale Amblyopie mit centralem Scotom ausgebildet, die unter geeigneter Behandlung eine Besserung erfahren hatte, aber nie vollständig gehoben war. Da der ophthalmoskopische Befund normal gewesen war, namentlich keine Veränderungen an der Macula lutea oder im Aussehen der Papillen bestanden hatten, so musste vor der genauen Untersuchung der Sehnerven die Ursache der Amblyopie unerklärt bleiben, um so mehr weil bis dahin in der Literatur kein Befund mitgetheilt war, der zur Erklärung des Falles hätte herangezogen werden können. Herr Professor Westphal hatte deshalb auch bei der kurz vor dem Tode erfolgten klinischen Vorstellung des Kranken besonders hervorgehoben, dass das centrale Scotom für die Diagnose nicht zu verwerthen sei, da es schon so lange bestanden nnd seine Ursachen bisher unbekannt seien. Auch nach dem Tode liess sich die in der linken Hemisphäre gefundene Herderkrankung als Grundleiden für die Scotome ausschliessen, da bei Affectionen in der Gegend der motorischen Rindenzone und des Centrum semiovale (31) bisher keine Sehstörungen, namentlich keine centralen Scotome beobachtet waren; die Gegend der Occipitalrinde war vollständig normal befunden. Als Ursache des Scotoms ergab nun die mikroskopische Untersuchung eine partielle Atrophie der Sehnerven ron der 
Papille bis ins Chiasma und den erhaltenen rechten Tractus - bei der grossen Symmetrie des Herdes in beiden Sehnerven und dem Chiasma hinsichtlich Grösse und Lage lässt sich mit Sicherheit annehmen, dass in dem linken Tractus derselbe Befund gewesen wäre. Entsprechend der bei der Untersuchung von Gesichtsfeldern gemachten Beobachtung, dass die Macula sowohl von Fasern des gekreuzten wie des nicht gelireuzten Fascieulus versorgt werden müsste, fand sich in dem Tractus in der Gegend des Fasciculus cruciatus und non cruciatus je ein gesonderter atrophischer Herd, während sich im Chiasma die zusammengehörigen Fasern bereits vereinigt hatten und in den Sehnervenstämmen bis in die Retina beisammen verliefen. Die die Maculagegend versorgenden Sohnervenfasern liegen nach diesem Befunde im Tractus am ventralen Rande und im obern äussern Quadranten in zwei von einander getrennten Bezirken, im Chiasma dicht unterhalb des Bodens des Recessus opticus, bleiben dort anch immer mehr in der dorsalen Hälfte und verlanfen in dem intracraniellen Abschnitt der Sehnerven bis zum Foramen opticum ziemlich genau central; ron hier abwärts ändern sie ihr Lageverhältniss unter gleichzeitiger Verinderung der Form der Bündelgruppe. Während sie vorher ein liegendes 0val darstellen, bilden sie zunächst in der Orbita ein mehr stehendes Oval, fast eine sichelförmige Figur, die urmittelbar hinter dem Foramen opticum nicht genau central-, sondern mehr temporalwärts gelegen ist. Nun bleiben sio auf der temporalen Seite, erreichen schliesslich beim Eintritt der Centralgefässe in den Sehnerven den temporalen Rand und verlaufen bis in die Papille hinein fast genau im nntern äussern Sector des Opticusquerschnitts in Gestalt eines Tails, dessen Basis der Rand des Sohnerven, 
dessen Spitze die Stelle der Centralgefässe bildet. In dieser Hinsicht ist der Fall also ein schönes Seitenstück zu dem von Samelsohn mitgetheilten Fall; durch denselben werden seine Angaben über den Verlauf und die Lage der Maculafasern in dem orbitalen Abschnitt des Sehnerven bis zur Papille auf's Glänzendste bestätigt. An meinem Präparat liess sich nur noch die Atrophie weiter hinauf nach dem Centrum zu verfolgen und dadurch tunsere Kenntniss über den Verlauf der Maculafasern bedeutend erweitern. - Als Grundleiden ergab sich vom Canalis opticus an bis abwärts zur Papille eine partielle Atrophie infolge einer Neuritis, die sich noch dureh die dichte Kerninfiltration, die Bindegewebswucherung der Septen, den Gefässreichthum nnd den Schwund der Sehnervenfasern manifestirte. Vom Canalis optiens aufwärts war eine atrophische Degeneration der Maculafasern obne Zeichen einer Fntzündung, sicher infolge der langjährigen Leitungstuterbrechung derselben im intraorbitalen $\mathrm{Ab}$ schnitt, eingetreten. Interessant ist dann noch in meinem Fall gewesen, dass sich im ganzen Verlauf der atrophischen Zone vom Tractus durch das Chiasma bis in die Retina Amyloidkörperehen in stellenweise auffallender Reichliehkeit nachweisen liessen, während sie bisher in der Netzhaut noch nie aufgefunden und beschrieben waren. - Ob der mässige Abusus spirituosorum in diesem Fall die Erkrankung der Optici herbeigeführt hatte, lasse ich dahingestellt, jedenfalls halte ich einen Zusammenhang in dem von Samelsohn gegebenen Sinne nicht für unmöglich.

Zum Schluss spreche ich noch Herrn Professor Leber für die freundliche Ueberlassung des vorliegenden Materials und Herm Professor Westphal für seine gütige Auskunft uber einige Punkte der Krankengesehichte meinen ergebensten Dank. - 


\section{Benutzte Literatur.}

1. Biesiadecki, Ueber das Chiasma nervorum opticorum des Menschen and der Thiere. Wiener academ. Sitzungsberichte. Math.-naturw. Kl. Bd. 42.

2. Michel, Ueber den Bau des Chiasma nervor. optic. Graefe's Archiv Bd. 19 Heft 2 1873. S. 59-86.

3. Mandelstamm, Ueber Sehnervenkreuzung und Hemiopic. Graefe's Archiv Bd. XIX. Heft 2, S. 39-58.

4. J. Müller, Zur vergleichenden Physiologie des Gesichtssinnes etc. Leipzig 1826. S. 114-141.

5. Hannover, Ueber den Bau des Chiasma opticum ete. Leiprig 1852.

6. Henle, Handbuch der Nervenlehre. 2. Lieferung, 1873.

7. v. Gudden, Ueber die Kreuzung der Nervenfasern im Chiasma nervorum opticorum. Archiv für Ophthalmologie v. Graefe, Bd. XX. 2, S. 249-268;

ferner Bd. XXI. Heft 3, S. 199-205;

ferner Bd. XXV. Heft 1, S. $1-56$ und Heft 4 , S. $237-246$.

8. Stilling, Untersuchungen über den Bau der optischen Centralorgane. Theil I, 1882.

9. Nicati, Archives de physiologie. 1878.

10. Munk, Vorträge in der Berliner physiol. Gesellschaft in den Jahren 1877, 1878, 1879 u. 1880 und am 3. Juni 1880 in der Berliner Akademie der Wissensehaften.

11. Marchand, Beitrag zur Kenntniss der homonymen bilateralen Hemianopsie u. s. w. Graefe's Arch. Bd. XXVIII. Heft 2, S. 63-96.

12. Woin ow, Bericht über den Heidelberger Ophthalmologencongress 1875. Zehender's Monatsblätter.

13. Purtscher, Veber Kreuzung und Atrophie der Nervi und Tractus optici. Graefe's Archiv Bd. XXVI. Heft 2, S. $191-220$.

14. Kellermann, Ausserordentliches Beilageheft zu Zehender's Monatsblättern, Jahrgang XVII.

15. Ganser, Ueber die periphere und centrale Anordnung der Sehnervenfasern u. s. w. Arehiv für Psychiatrie Bd. XIII. Heft 2, S. $341 \mathrm{ff}$.

16. Leber, Ueber das Vorkommen von Anomalien des Farbensinnes ete. Graefe's Archiv Bd. XV. Heft 3, 
S. 26-107 und im Handbuch von Graefe-Sämisch, Krankheiten der Netzhaut and des Selnerven.

17. Samelsohn, Zur Anatomie und Nosologie der retrobulbären Neuritis u. s. w. Graefe's Archiv Bd. XXVIII. Heft 1, p. 1-110.

18. Liebreich, Klinische Monatsblätter von Zchender, Jahrgang VII, 1869.

Ueber den Vorlauf dor Nervenfasern auf der Papille und in der Rotina. Sitzungsbericht der Ophthalmolog. Gesellschaft.

19. Michel, Ueber die Ansstrahlungsweise der Optieusfasern in der menschlichen Retina. Leipzig 1875.

Beiträge zur Anatomie und Plysiologie. Jubelschrift, C. Ludwig gewidmet.

20. Schwalbe, Mikroskopische Anatomie des Sehnerven in Graefe-Sämisch Bd. 1.

21. Magnus, Die Sehnervenblutungon. Leipzig 1874.

22. Wilbrand, Ueber Nemitis axialis, Zolender's Monatsblätter, Jahrgang 1878.

23. Nettleship, Transact. of the Ophthal. Societ. Vol. I.

24. Binswanger, Ueber die Beziehungen der sog. motorischen Rindenzone u. s. w. Arehiv für Psyehiatrio Bd. XI. Heft 3.

25. Schiefferdecker, Veber die Verwendung des Celloidins u. s. w. Archiv für Anatomie und Entwickelungsgeschichte, Jahrg. 1882, Heft 2 und 3.

26. Mihalkovicz; und Michel, efr. Graefe's Archiv XIX. Heft 2, S. 77 ff. (1873).

27. Lusehka, Anatomie des Kopfes p. 505.

28. Michel, Ueber eine Hyperplasio des Chiasma u. s. w. Graefe's Archiv Bd. XIX. Heft 3, S. 145-164.

29. Leber, Ueber ein eigenthümliehes Verhalten der Corpuscula amylacea u. s. w. Graefe's Arehiv Bd. XIX. Heft 1, S. 191-202 und Bd. XXV. Heft 1.

30. Treitel, Beiträge zur patholog. Anatomie des Auges. Graefe's Archiv Bd. XXII. Heft 2.

31. Nothnagel, Topische Diagnostik der Hirnkrankheiten; Wernicke, Lehrbuch der Gehirnkrankheiten; Charcot, tocalisations dans les maladies du cerverau. 


\section{Erklärung der Abbildungen.}

Fig. 1. Vergrösserung \%. Horizontalschnitt dureh die 1. Papille und den hintern Bulbusabsehmitt.

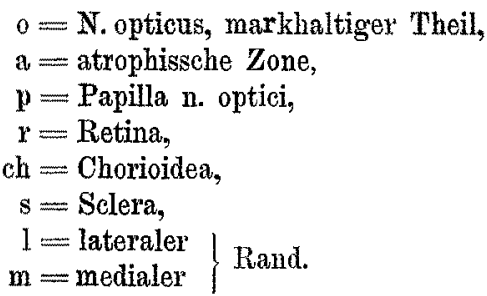

Fig. 2. Vergrösserung $\%$, Querschnitt durch den 1 . Opticus dicht am Bulbus, von vorn gesehen.

$$
\begin{aligned}
\left.\begin{array}{rl}
\mathrm{l}= & \text { lateraler } \\
\mathrm{m}= & \text { medialer } \\
0 & =\text { oberer } \\
\mathrm{u} & =\text { unterer }
\end{array}\right\} \text { Rand, } \\
\mathrm{a}=\text { die helle atrophische Stelle im untern äussern } \\
\quad \text { Quadranten mit der Spitze nach den Central- } \\
\quad \text { gefässen (c) und der Basis nach der Pial- } \\
\quad \text { seheide (p), } \\
\mathrm{d}=\text { Duralscheide, gefaltet, } \\
\mathrm{J}=\text { verbreiterter Intervaginalraum. }
\end{aligned}
$$
Quadranten mit der Spitze nach den Centralgefässen (c) und der Basis nach der Pial-

Fig. 3. Vergrösserung \%. Quersehnitt durch den 1. Opticus, $8 \mathrm{~mm}$ hinter dem Bulbus von vorn gesehen, die helle Stelle ist der atrophische Herd. $\mathrm{m}, \mathrm{l}, \mathrm{o}, \mathrm{u}$ wie zuvor.

Fig. 4. Vergrösserung \% Querschnitt dureh den 1. Opticus, $16 \mathrm{~mm}$ hinter dem Bulbus vor der Eintrittsstelle der Gefässe, von vorn gesehen. Bezeichnung wie zuvor. Die helle Stelle ist der atrophische Herd.

Fig. 5. Vergrösserung \%. Querschnitt durch den 1. Opticus, $24 \mathrm{~mm}$ hinter dem Bulbus, vor dem Canalis opticus, voll vorn gesehen. Bezeichnung wie zuvor; die helle Stelle ist der atrophische Herd.

Fig. 6. Querschnitt durch den 1. N. opticus, dicht hinter dem Canalis opticus. Vergrösserang $\%$, von vorn gesehen. Bezeichnung wie zovor, die helle Stelle ist der atrophische Herd.

Fig. 7. Querschnitt durch den 1 . Opticus ca. $1 \mathrm{~cm}$ vor dem Chiasma, von vorn gesehen. Vergrösserung \%. Bezeichnung wie zavor. Die helle Stelle in der Mitte ist dex atrophische Herd, die feinen weissen Striche und der Streifen vom obern Rande sind verdickte Septen. 
Fig. 8. Frontalschnitt duruh das Chiasma in der vordern Hälfte, von vorn gesehen. Vergrösserung $\%$. R rechte, L linke Hälfte. Unter d (dorsaler Rand) ein heller Strich feinkörnig-faseriger Substanz und erst unter diesem der atrophische Herd a. p Piauberzug anf der ventralen Fläche mit dem Anfang der Hypophysis.

Fig. 9. Frontalsehnitt durch das Chiasma in der hintern Hälfte, von vorn gesehen. Vergrösserung $\%$ Recessus opticus $-\mathrm{R} 0-$ getroffen; derselbe liegt eingebettet in grauer Substanz, unter dieser der atrophische Herd a. R. and $\mathrm{L}$. wie zuvor.

Fig. 10. Frontalschnitt durch den rechten Tractus opticus, von vorn gesehen. Vergrösserung \%.

T $c=$ Tuber cinereum mit grossen Vacuolen für Gefässquerschnitte,

$T_{0}=$ Tractus opticus,

$\mathrm{a} b=$ atrophische Stellen am untern und obem Rande

$c=$ normale Substanz.

Fig. 11. Querschnitt durch den 1.Opticus, entsprecheud dem Sehnitt Fig. 5, von vorn gesehen. Vergrösserung $T_{1}$ zux besseren Veranschaulichung des atrophischen Herdes.

Fig. 12. Querschnitt durch den 1. Opticus, entsprechend der Fig. 2, dicht am Bulbus, von vorn gesehen. Vergrösserung $1 \%$. Bezeichnung wie dort.

$\mathrm{a}=$ atropischer Herd, $c=$ Centralgefässe.

Hig. 13. Querschnitt durch den l. Opticus, dieht hinter dem Fornu. opticum, cerebralwärts. Vergrösserung 10\%. Bezeichnung wie zuror.

Fig. 14. Querschnitt durch den rechten Tractus, entsprechend Figur 10, von vorn gesehen. Vergrösserung $\%$. Die dunklen Striche sind Septen mit Gefässen.

$\mathrm{a} a \cdot \mathrm{b}=$ atrophische Herde im Fasciculus cruciatus und noncruciatus,

$c=$ normale Substanz des Tractus,

d = ein grösserer Gefässdurchschnitt an der Grenze gegen das Tuber cinereum (T. c.), go = Gangl. optic. mit grösseren Gefässvacuolen, To $=$ Tractus opticus.

Fig. 15. Papillèndurchschnit, entsprechend Figux 1. Vergrösserung $\mathrm{x} / \mathrm{s}$.

$$
\left.\begin{array}{rl}
a & =\text { atrophische Stelle } \\
c & =\text { Centralgefässs, } \\
1 & =\text { temporaler } \\
m & =\text { nasaler }
\end{array}\right\} \text { Retinaabschnitt. }
$$



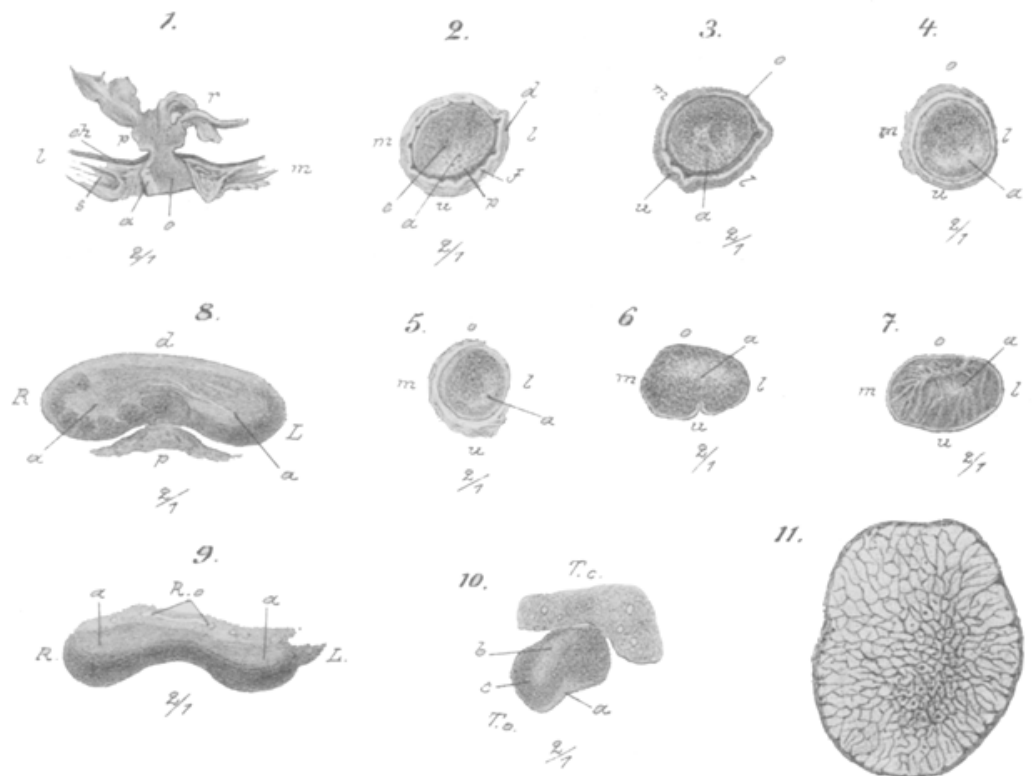

II.

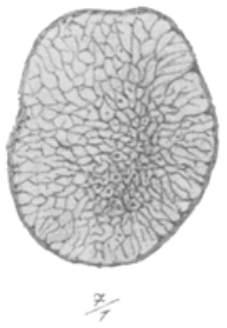

13.
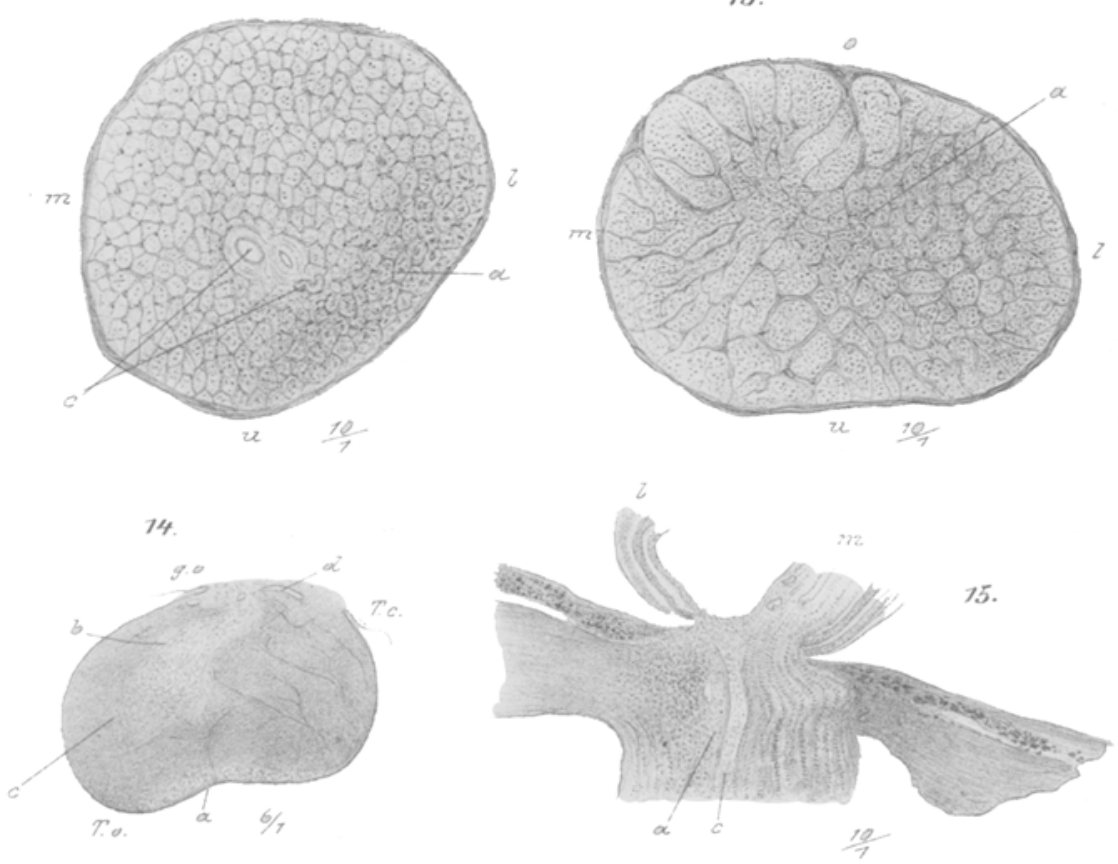

Granue for. Alb. Sanntze Sith. Bnost. Beation. 